\title{
On Fuzzy Proper Exact Sequences and Fuzzy Projective Semimodules Over Semirings
}

\author{
AMARJIT KAUR SAHNI ${ }^{1}$, JAYANTI TRIPATHI PANDEY ${ }^{2}$, \\ RATNESH KUMAR MISHRA ${ }^{3}$, VINAY KUMAR ${ }^{4}$ \\ ${ }^{1,2}$ Department of Mathematics, AIAS, Amity University, Uttar Pradesh, INDIA \\ ${ }^{3}$ Department of Mathematics, NIT, Jamshedpur, INDIA \\ ${ }^{4}$ Ex Scientist GOI, Ex Dean, Professor VSIT, GGSIP University, Delhi, INDIA
}

\begin{abstract}
As an analogue here we extend and give new horizon to semimodule theory by introducing fuzzy exact and proper exact sequences of fuzzy semi modules for generalizing well known theorems and results of semimodule theory to their fuzzy environment. We also elucidate completely the characterization of fuzzy projective semi modules via Hom functor and show that semimodule $\mu_{P}$ is fuzzy projective if and only if $\operatorname{Hom}\left(\mu_{P},-\right)$ preservers the exactness of the sequence $\mu_{M^{\prime}} \stackrel{\bar{\alpha}}{\rightarrow} \nu_{M} \stackrel{\bar{\beta}}{\rightarrow} \eta_{M^{\prime \prime}}$ with $\bar{\beta}$ being K-regular. Some results of commutative diagram of R-semimodules having exact rows specifically the "5-lemma" to name one, were easily transferable with the novel proofs in their fuzzy context. Also, towards the end apart from the other equivalent conditions on homomorphism of fuzzy semimodules it is necessary to see that in semimodule theory every fuzzy free is fuzzy projective however the converse is true only with a specific condition.
\end{abstract}

Key-words:- fuzzy semimodules, fuzzy projective module, fuzzy projective semimodule, 5-lemma,fuzzy exact sequence, fuzzy proper exact sequence.

Received: July 20, 2021. Revised: November 27, 2021. Accepted: December 18, 2021. Published: December 27, 2021.

\section{Introduction}

From 1965 onwards when the crucially relevant concept "fuzzy" came into existence, number of structures in algebra were extended to their fuzzy versions[21]. After which researchers did everything they could, to clarify concepts in the field of fuzzy module theory, leaving no stone unturned[3], [4], [7], [8], [12], [22]. And here in this paper we primarily deals with two aspects mainly: the study of fuzzy semimodules and fuzzy projective semimodules over semirings, where semiring is a structure near to ring but apart from the necessary condition of having an additive inverse. The term semiring was first coined by Vandiver[19], after which the concepts of automata and formal languages in[9] and [5] was extensively studied in its fuzzy context in which semirings act as a vital tool. Shu and Wang [16], [17] discussed the cardinality of bases and dimensional formu- las of semimodules over commutative semirings. In the light of forgoing here we study the fuzzy context of semimodules, free and projective semimodules over semirings in order to set a new platform for future researches.

The present study is structured as follows. In Section 2 along with basic definitions appropriate examples have been constructed to support the study. In section 3 we have investigated and generalized the concept of semimodules and proved many interesting results. Section 4 analyses the concept of fuzzy projective semimodules. In it generalization from the corresponding results of the classical theory, along with the equivalent description of the same with a particular condition has being mentioned. At last, section 5 discusses the applications and future scope of the current study. 


\section{Preliminaries}

Definitions and results applied during the present study are discussed below.

Terminology used extensively in this study :

1. $\mathrm{R}$ is a ring that has its identity.

2. pd means projective dimension

3. $\exists$ means there exists

4. Fuzzy module over the module $M$ is denoted as $\mu_{M}$.

5. $\Rightarrow$ means implies

6. $\mu(m)$ represents the arbitrary element of fuzzy set $\mu_{M}$.

Definition 1. [1] An additively written commutative semigroup $M$ with a neutral element 0 is called a right $R$-semimodule $M_{A}$, if $R$ is a semiring and there is a function $\alpha: M x R \rightarrow M$ such that if $\alpha(m, a)$ is denoted by ma then the following conditions hold:

(i) $\left(m+m^{\prime}\right) a=m a+m^{\prime} a$,

(ii) $m\left(a+a^{\prime}\right)=m a+m a^{\prime}$,

(iii) $m\left(a a^{\prime}\right)=(m a) a^{\prime}$,

(iv) $m .1=m$,

(v) $0 . a=m .0=0$ for all $a, a^{\prime} \in R$ and $m, m^{\prime} \in$ $M$.

Definition 2. [1] Let $M$ be a right $R$ semimodule. A function $\lambda: M \rightarrow L$ is called a fuzzy subsemimodule of $M_{R}$, if the following conditions hold:

(i) $\lambda\left(0_{M}\right)=1$,

(ii) $\lambda\left(m+m^{\prime}\right) \geq \lambda(m) \wedge \lambda\left(m^{\prime}\right)$ for all $m, m^{\prime} \in$ $M$.

(iii) $\lambda(m a) \geq \lambda(m)$ for all $m \in M$ and $a \in R$.

Remark 1. [1] : If $\bar{\pi}$ is a fuzzysubsemimodule of a right semimodule say $M_{R}$ then $\bar{\pi}\left(0_{R}\right)=1$ and in the sequel fuzzysubsemimodules of $M_{R}$ are called fuzzy right ideals of the semiring $R$.

Proposition 1. [1] : If $\bar{\pi}$ is a fuzzysubsemimodule of a right semimodule say $M_{R}$ and $\mu$ a fuzzy ideal of $R$ then $\bar{\pi} \mu$ is a fuzzysubsemimodule of $M$.

\section{$3 \quad$ Fuzzy Subsemimodules}

NOTE : For notational convenience we will call fuzzy subsemimodules as fuzzy semimodules.
In this section $\mathrm{R}$ represents the semiring having identity 1 and each semimodule $M$ is unitary complying $1 . x=x$ for all $x \in M$. Here we have characterized fuzzy semimodules and were able to establish interesting connecting theorems, propositions and results.

Example 1. Let $Z_{0}$ be a semiring where it denotes set of integers that are not negative. Also, $Z$ denote the set of integers forming a semimodule over $Z_{0}$. Then a map $\bar{\pi}: Z \rightarrow[0,1]$ defined as

$$
\bar{\pi}(z)=\left\{\begin{array}{l}
1, \text { if } z=0 \\
0.8, \text { if } z \neq 0
\end{array}\right.
$$

forms a fuzzysubsemimodule as it satisfies definition 2 as shown below:

(i) By definition of $\bar{\pi}$

(ii) True as presented in the following figures: For any value of $Z$ say $0,1,2,3,4,5$ we have

\begin{tabular}{|c|c|c|c|c|c|c|}
\hline & 0 & 1 & 2 & 3 & 4 & 5 \\
\hline \multirow{2}{*}{0} & 1 & 0.8 & 0.8 & 0.8 & 0.8 & 0.8 \\
\hline & 0.8 & 0.8 & 0.8 & 0.8 & 0.8 & 0.8 \\
\hline$?$ & 0.8 & 0.8 & 0.8 & 0.8 & 0.8 & 0.8 \\
\hline & 0.8 & 0.8 & 0.8 & 0.8 & 0.8 & 0.8 \\
\hline 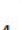 & 0.8 & 0.8 & 0.8 & 0.8 & 0.8 & 0.8 \\
\hline 5 & 0.8 & 0.8 & 0.8 & 0.8 & 0.8 & 0.8 \\
\hline
\end{tabular}

Fig.1 Working of "+" operation on the elements of Z

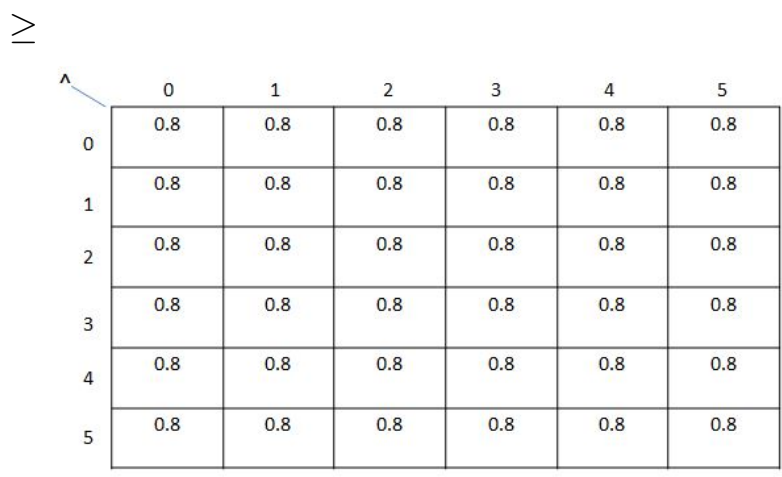

Fig.2 Working of " $\wedge$ " operation on the elements of $\mathrm{Z}$

(iii) For instance let $m \in Z$ be 4 and $a \in Z_{0}$ be 8 then $\lambda(m a) \geq \lambda(m)$ holds true by the definition of $\bar{\pi}$ since $0.8 \geq 0.8$. Likewise for any other value of $Z$ and $Z_{0}$ the mentioned condition is satisfied. Thus, $\bar{\pi}$ can be termed as fuzzysubsemimodule. 
Example 2. Let $Z_{0}$ be a semiring where it represents the set of integers that are not negative. Let $Q_{0}$ denote the set of non negative rational numbers forming a semimodule over semiring $Z_{0}$ then a map $\bar{\nu}: Q_{0} \rightarrow[0,1]$ defined as

$$
\nu(q)=\left\{\begin{array}{l}
1, \text { if } q=0 \\
0.7, \text { if } q \neq 0
\end{array}\right.
$$

forms a fuzzysubsemimodule. The same can be exhibited in the similar way as was in example 1. Definition 3. Let $\bar{f}: \mu_{M} \rightarrow \nu_{N}$ be the homomorphism of fuzzy semimodules. Then $\operatorname{Im} \bar{f}$ of $\nu_{N}$ is defined as $\operatorname{Im} \bar{f}=\left[\nu(n) \in \nu_{N}: \nu(n)+\bar{f}\left(\mu\left(m_{1}\right)\right)\right.$ $=\bar{f}\left(\mu\left(m_{2}\right)\right)$ for some $\mu\left(m_{1}\right)$ and $\left.\mu\left(m_{2}\right) \in \mu_{M}\right]$. And, $\operatorname{Ker} \bar{f}=\left\{\mu(m) \in \mu_{M}: \bar{f}(\mu(m))=0_{\nu_{N}}\right\}$.

Definition 4. Let $\bar{f}: \mu_{M} \rightarrow \nu_{N}$ be the homomorphism of fuzzy semimodules. Then $\bar{f}$ is said to be i-regular if $\bar{f}\left(\mu_{M}\right)=\operatorname{Im} \bar{f}$. And K-regular if $\bar{f}\left(\mu\left(m_{1}\right)\right)=\bar{f}\left(\mu\left(m_{2}\right)\right)$ implies $\mu\left(m_{1}\right)+\mu\left(m_{3}\right)$ $=\mu\left(m_{2}\right)+\mu\left(m_{4}\right)$ for some $\mu\left(m_{3}\right)$ and $\mu\left(m_{4}\right) \in$ $K e r \bar{f}$. Also, the same is termed as fuzzy semimonomorphism if $\operatorname{Ker} \bar{f}=0$.

Definition 5. Let the sequence of fuzzy semimodules and fuzzy semimodule homomorphisms

$\ldots . \rightarrow \mu_{n-1} \stackrel{f_{n-1}^{-}}{\longrightarrow} \mu_{n} \stackrel{\bar{f}_{n}}{\longrightarrow} \mu_{n+1} \rightarrow \ldots$ is termed as fuzzy exact if $\operatorname{Im} f_{n-1}^{-}=\operatorname{Ker} \bar{f}_{n}$ for each $n$. And is called as proper exact if $f_{n-1}^{-}\left(\mu_{n-1}\right)=K e r \bar{f}_{n}$ for every $n$.

Definition 6. The exact sequence of fuzzy semimodules of the form $0 \rightarrow \mu_{A} \stackrel{\bar{f}}{\rightarrow} \eta_{B} \stackrel{\bar{g}}{\rightarrow} \nu_{B} \rightarrow 0$ is called as the fuzzy short exact sequence. And the same is termed as fuzzy split if for some $\bar{h} \in \operatorname{Hom}\left(\eta_{B}, \mu_{A}\right)$ we have $\bar{f} \bar{h}=i d_{\mu_{A}}$ for some fuzzy semimodules $\eta_{B}$ and $\mu_{A}$.

Definition 7. Assuming $N$ to be $R$-semimodule and $\nu_{N}$ be fuzzy semimodule over it. Then the set $n_{1}, n_{2}, \ldots, n_{m} \in N$ is linearly independent with regard to $\nu_{N}$ if it meets the following two criteria

(i) The set $\left[n_{1}, n_{2}, \ldots, n_{m}\right]$ is linearly independent,

(ii) $\nu\left(a_{1}+a_{2}+\ldots+a_{m}\right)=\min \left[\nu\left(a_{1}\right), \ldots\right.$, $\left.\nu\left(a_{m}\right)\right]$ for any $a_{i} \in \mathrm{R} n_{i}, 1 \leq i \leq \mathrm{m}$.

Also, it is said that a subset $B$ of $N$ in definition 7 is called fuzzy pseudo basis of $\nu_{N}$ if $B$ is maximal subset of $N$ that is any finite subset $\left[n_{1}, n_{2}, \ldots ., n_{k}\right]$ of $B$ is linearly independent. And, the fuzzy pseudo basis of $\nu_{N}$ is also referred as a fuzzy basis for $N$ in this context.

Lemma 1. Let $R$ be a semiring, then the sequence of fuzzy R-semimodules $\mu_{M^{\prime}} \stackrel{\bar{\alpha}}{\rightarrow} \nu_{M} \stackrel{\bar{\beta}}{\rightarrow} \eta_{M^{\prime \prime}}$ is fuzzy exact if there exists fuzzy exact nonhorizontal sequences in the following diagram.

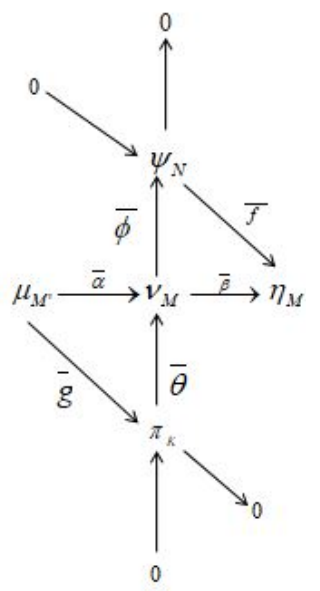

Fig.3 Representing fuzzy exact non-horizontal sequences

Proof. Let $\nu(m) \in \operatorname{Ker} \bar{\beta}$. Also,

$$
\begin{aligned}
\bar{f} \bar{\phi} & =\bar{\beta} \\
\bar{f} \bar{\phi}(\nu(m)) & =\bar{\beta}(\nu(m)) \\
& =0
\end{aligned}
$$

implies $\bar{\phi}(\nu(m)) \in \operatorname{Ker} \bar{f}$. Since Ker $\bar{f}=0$ then $\nu(m) \in \operatorname{Ker} \bar{\phi}=\operatorname{Im} \bar{\theta} . \quad \nu(m)+\bar{\theta}\left(\pi\left(k_{1}\right)\right)$ $=\bar{\theta}\left(\pi\left(k_{2}\right)\right)$. Implies $\left[\nu(m)+\bar{\theta} \bar{g}\left(\mu\left(m_{1}^{\prime}\right)\right]=\right.$ $\bar{\theta} \bar{g}\left(\mu\left(m_{2}^{\prime}\right)\right.$. And $\nu(m)+\bar{\alpha}\left(\mu\left(m_{1}^{\prime}\right)=\bar{\alpha}\left(\mu\left(m_{2}^{\prime}\right)\right.\right.$ since $\bar{\theta} \bar{g}=\bar{\alpha}$. Thus, $\nu(m) \in \operatorname{Im} \bar{\alpha}$.

Conversely, let $\nu(m) \in \operatorname{Im} \bar{\alpha} . \nu(m)+\bar{\alpha}\left(\mu\left(m_{1}^{\prime}\right)\right.$ $=\bar{\alpha}\left(\mu\left(m_{2}^{\prime}\right)\right.$. Again since $\bar{\theta} \bar{g}=\bar{\alpha}$ then, $[\nu(m)+$ $\bar{\theta} \bar{g}\left(\mu\left(m_{1}^{\prime}\right)\right]=\left[\bar{\theta} \bar{g}\left(\mu\left(m_{2}^{\prime}\right)\right]\right.$ implying $\nu(m) \in \operatorname{Im} \bar{\theta}$. But $\operatorname{Im} \bar{\theta}=\operatorname{Ker} \bar{\phi}$. Hence $\bar{f} \bar{\phi}=\bar{\beta} . \bar{f} \bar{\phi} \nu(m)=$ $\bar{\beta} \nu(m)$ gives $\bar{\beta} \nu(m)=0$ thus, $\nu(m) \in \operatorname{Ker} \bar{\beta}$.

Result 1. For fuzzy semimodules every proper exact sequence is fuzzy exact.

Proof. $\mu_{M} \stackrel{\bar{\alpha}}{\rightarrow} \nu_{N} \stackrel{\bar{\beta}}{\rightarrow} \eta_{M^{\prime}}$ be the fuzzy proper exact sequence. So we have $\operatorname{Ker} \bar{\beta}=\bar{\alpha}\left(\mu_{M}\right) \subseteq \operatorname{Im} \bar{\alpha}$. 
Thus, we are left to show $\operatorname{Im} \bar{\alpha} \subseteq \operatorname{Ker} \bar{\beta}$. Let $(\nu(n))$ $\in \operatorname{Im} \bar{\alpha}$ so we have $(\nu(n))+\bar{\alpha}\left(\mu\left(m_{1}\right)\right)=\bar{\alpha}\left(\mu\left(m_{2}\right)\right)$ which implies $(\nu(n)) \in \bar{\alpha}\left(\mu_{M}\right)=\operatorname{Ker} \bar{\beta}$. Hence the given sequence is fuzzy exact.

Corollary 1. For a semiring $R$, the sequence of fuzzy R-semimodules $\mu_{M^{\prime}} \stackrel{\bar{\gamma}}{\rightarrow} \nu_{M} \stackrel{\bar{\beta}}{\rightarrow} \eta_{M^{\prime \prime}}$ is fuzzy proper exact if the non-horizontal sequences of the following commutative diagram are fuzzy proper exact.

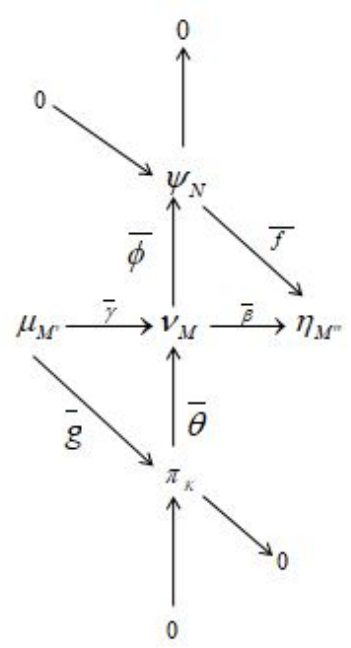

Fig.4 Illustrating fuzzy proper exact non-horizontal sequences

Proof. By above result and lemma $1, \operatorname{Ker} \bar{\beta}=\mathrm{Im}$ $\bar{\gamma}$, hence $\bar{\gamma}\left(\mu_{M^{\prime}}\right) \subset \operatorname{Ker} \bar{\beta}$, Now let $\nu(m) \in \operatorname{Ker}$ $\bar{\beta}$ then $\bar{\beta}(\nu(m))=\bar{f} \bar{\phi}(\nu(m))=0$ hence $\bar{\phi}(\nu(m))$ $\in \operatorname{Ker} \bar{f}$. But $\operatorname{Ker} \bar{f}=0$, therefore $\nu(m) \in \operatorname{Ker} \bar{\phi}$ $=\bar{\theta}\left(\pi_{K}\right)$. Hence $\nu(m)=\bar{\theta}(\pi(\mathrm{k}))=\bar{\theta}\left(\bar{g} \mu\left(m^{\prime}\right)\right)=$ $\bar{\gamma} \mu\left(m^{\prime}\right)$. Hence $\bar{\gamma} \mu_{M}=\operatorname{Ker} \bar{\beta}$.

Corollary 2. For a semiring $R$, the sequence of fuzzy $R$-semimodules with $\bar{\beta}$ being K-regular $\mu_{M^{\prime}} \stackrel{\bar{\alpha}}{\rightarrow} \nu_{M} \stackrel{\bar{\beta}}{\rightarrow} \eta_{M^{\prime \prime}}$ is fuzzy proper exact if the nonhorizontal sequences of the following commutative diagram are fuzzy proper exact.

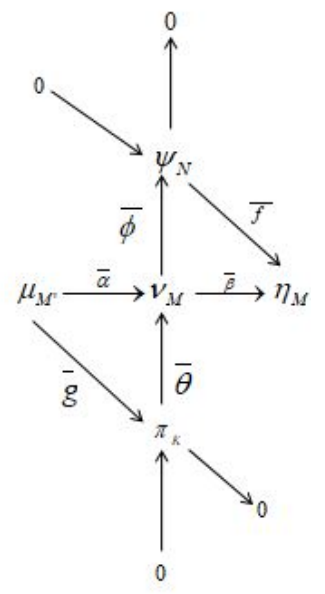

Fig.5 Symbolizing fuzzy proper exact sequence with $\bar{\beta}$ being K-regular

Proof. For $\bar{\beta}$ being K-regular let $\mu_{M^{\prime}} \stackrel{\bar{\alpha}}{\rightarrow} \nu_{M} \stackrel{\bar{\beta}}{\rightarrow} \eta_{M^{\prime \prime}}$ be a fuzzy proper exact sequence. Consider the following diagram :

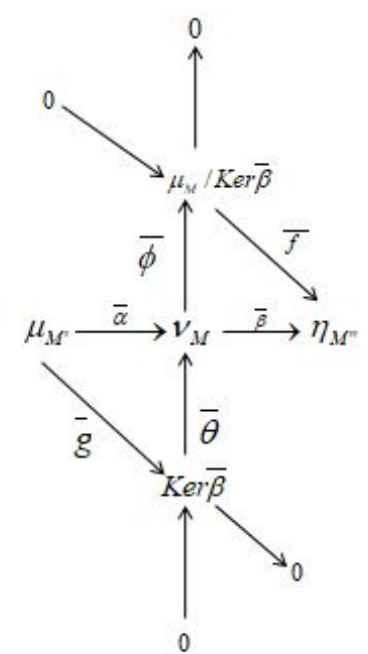

Fig.6 Fuzzy proper exact sequence

where $\bar{g}\left(\mu\left(m^{\prime}\right)\right)=\bar{\alpha}\left(\mu\left(m^{\prime}\right)\right)$ for all $\mu\left(m^{\prime}\right) \in \mu_{M^{\prime}}$, $\bar{\theta}(\nu(m))=\nu(m)$ for all $\nu(m) \in \operatorname{Ker} \bar{\beta}, \bar{\phi}(\nu(m))=$ $\nu(m) / \operatorname{Ker} \bar{\beta}$ for all $\nu(m) \in \nu_{M}$ and $\bar{f}(\nu(m) /$ ker $\bar{\beta})=\bar{\beta}(\nu(m))$ for all $\nu(m) / \operatorname{ker} \bar{\beta} \in \nu_{M} / \operatorname{Ker} \bar{\beta}$. Let $\nu\left(m_{1}\right) / \operatorname{Ker} \bar{\beta}=\nu\left(m_{2}\right) / \operatorname{Ker} \bar{\beta}$ then $\nu\left(m_{1}\right)$ $+\nu\left(m_{3}\right)=\nu\left(m_{2}\right)+\nu\left(m_{4}\right) \in \operatorname{Ker} \bar{\beta}$. Hence $\bar{\beta} \nu\left(m_{1}\right)=\bar{\beta} \nu\left(m_{2}\right)$ therefore $\bar{f}$ is well defined. Now If $\bar{\beta} \nu\left(m_{1}\right)=\bar{\beta} \nu\left(m_{2}\right)$ we have $\nu\left(m_{1}\right)+\nu\left(m_{3}\right)$ $=\nu\left(m_{2}\right)+\nu\left(m_{4}\right)$ where $\nu\left(m_{3}\right)$ and $\nu\left(m_{4}\right) \in$ $\operatorname{Ker} \bar{\beta}$ as $\bar{\beta}$ is K-regular. Hence $\nu\left(m_{1}\right) / \operatorname{Ker} \bar{\beta}=$ $\nu\left(m_{2}\right) /$ Ker $\bar{\beta}$ implying $\bar{f}$ is injective. Therefore, 
the sequence $0 \rightarrow \operatorname{Ker} \bar{\beta} \stackrel{\bar{\theta}}{\rightarrow} \nu_{M} \stackrel{\bar{\phi}}{\rightarrow} \nu_{M} / \operatorname{ker} \bar{\beta} \rightarrow 0$ is fuzzy proper exact and the diagram above is commutative. Converse, can be easily derived from Corollary 1.

Corollary 3. For a semiring $R$, the sequence of fuzzy $R$-semimodules with $\bar{\beta}$ being K-regular $\mu_{M^{\prime}} \stackrel{\bar{\alpha}}{\rightarrow} \nu_{M} \stackrel{\bar{\beta}}{\rightarrow} \eta_{M^{\prime \prime}} \quad$ is fuzzy exact if the nonhorizontal sequences of figure 5 are fuzzy exact.

Proof. Let $\mu_{M^{\prime}} \stackrel{\bar{\alpha}}{\rightarrow} \nu_{M} \stackrel{\bar{\beta}}{\rightarrow} \eta_{M^{\prime \prime}}$ be a fuzzy proper exact sequence with $\bar{\beta}$ being K-regular. Consider the following diagram :

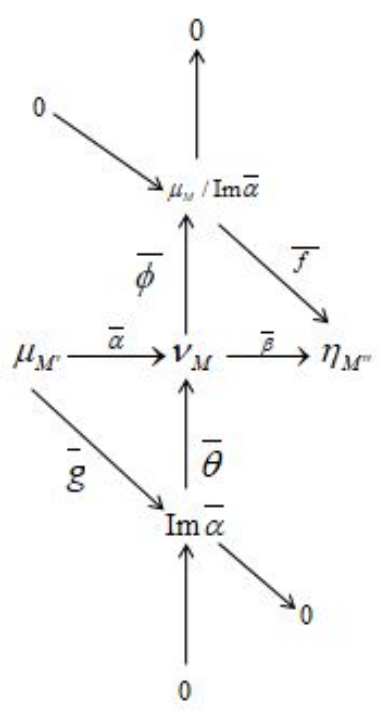

Fig.7 Symbolizing fuzzy exact sequence with $\bar{\beta}$ being K-regular

where $\bar{g}\left(\mu\left(m^{\prime}\right)\right)=\bar{\alpha}\left(\mu\left(m^{\prime}\right)\right) \in \operatorname{Im} \bar{\alpha}$ for all $\mu\left(m^{\prime}\right)$ $\in \mu_{M^{\prime}}, \bar{\theta}(\nu(m))=\nu(m)$ for all $\nu(m) \in \operatorname{Im} \bar{\alpha}$, $\bar{\phi}(\nu(m))=\nu(m) / \operatorname{Im} \bar{\alpha}$ for all $\nu(m) \in \nu_{M}$ and $\bar{f}(\nu(m) / \operatorname{Im} \bar{\alpha})=\bar{\beta}(\nu(m))$ for all $\nu(m) / \operatorname{Im} \bar{\beta} \in$ $\nu_{M} / \operatorname{Im} \bar{\alpha}$.

Let $\nu\left(m_{1}\right) / \operatorname{Im} \bar{\alpha}=\nu\left(m_{2}\right) / \operatorname{Im} \bar{\alpha}$ then $\nu\left(m_{1}\right)+$ $\nu\left(m_{3}\right)=\nu\left(m_{2}\right)+\nu\left(m_{4}\right) \in \operatorname{Im} \bar{\alpha}=\operatorname{Ker} \bar{\beta}$. Hence $\bar{\beta} \nu\left(m_{1}\right)=\bar{\beta} \nu\left(m_{2}\right)$ confirming $\bar{f}$ is well defined. Let If $\bar{\beta} \nu\left(m_{1}\right)=\bar{\beta} \nu\left(m_{2}\right)$ and as $\bar{\beta}$ is K-regular we have $\nu\left(m_{1}\right)+\nu\left(m_{3}\right)=\nu\left(m_{2}\right)+$ $\nu\left(m_{4}\right)$ where $\nu\left(m_{3}\right)$ and $\nu\left(m_{4}\right) \in \operatorname{Ker} \bar{\beta}=\operatorname{Im} \bar{\alpha}$. Hence $\nu\left(m_{1}\right) / \operatorname{Im} \bar{\alpha}=\nu\left(m_{2}\right) / \operatorname{Im} \bar{\alpha}$ therefore $\bar{f}$ is injective. Clearly, the sequence $0 \rightarrow \operatorname{Im} \bar{\alpha} \stackrel{\bar{\theta}}{\rightarrow} \nu_{M}$ $\stackrel{\bar{\phi}}{\rightarrow} \nu_{M} / \operatorname{Im} \bar{\alpha} \rightarrow 0$ is fuzzy exact. Therefore the above mentioned figure is commutative. Converse, can be easily derived from Lemma 1.
Result 2. Exact sequence $\mu_{M} \stackrel{\bar{\alpha}}{\rightarrow} \nu_{N} \stackrel{\bar{\beta}}{\rightarrow} \eta_{M^{\prime}} \quad$ of fuzzy semimodules having $\bar{\alpha}$ i-regular is fuzzy proper exact.

Proof. Since the given sequence is fuzzy exact $\operatorname{ker} \bar{\beta}=\operatorname{Im} \bar{\alpha}$. Now, $\bar{\alpha}\left(\mu_{M}\right) \subseteq \operatorname{Im} \bar{\alpha}=\operatorname{Ker} \bar{\beta}$ implies $\bar{\alpha}\left(\mu_{M}\right) \subseteq \operatorname{Ker} \bar{\beta}$. To show the converse let $\nu(n) \in$ $\operatorname{Ker} \bar{\beta}=\operatorname{Im} \bar{\alpha}$. Thus, $\nu(n) \in \operatorname{Im} \bar{\alpha}$ and since $\bar{\alpha}$ is $i$-regular $\nu(n) \in \bar{\alpha}\left(\mu_{M}\right)$.

Theorem 1. For a commutative diagram of fuzzy $R$-semimodules, having exact rows of $R$ - semimodule homomorphisms statements 1 and 2 holds true.

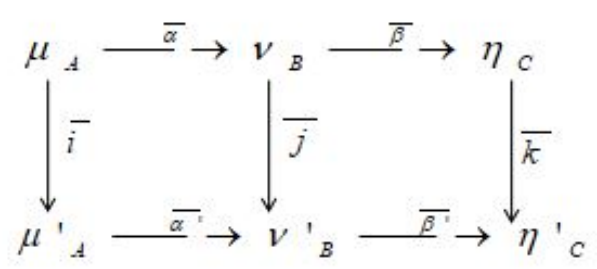

Fig.8 Commutative fuzzy exact semimodule homomorphisms

\section{NOTE FOR FIGURE 8}

(i). First Row is $\mu_{A} \stackrel{\bar{\alpha}}{\rightarrow} \nu_{B} \stackrel{\bar{\beta}}{\rightarrow} \eta_{C}$

(ii). Second Row is $\mu_{A}^{\prime} \stackrel{\bar{\alpha}^{\prime}}{\rightarrow} \nu_{B}^{\prime} \stackrel{\bar{\beta}^{\prime}}{\rightarrow} \eta_{C}^{\prime}$

(iii). First Row is fuzzy exact means $\operatorname{Im} \bar{\alpha}=$ Ker $\bar{\beta}$ and similarly for the second row.

(iv). Commutative diagram means $\bar{j} \bar{\alpha}=\bar{\alpha}^{\prime} \bar{i}$ and $\bar{k} \bar{\beta}=\bar{\beta}^{\prime} \bar{j}$

1. If $\bar{j}$ is injective and $\bar{i}, \bar{\beta}$ are surjective then $\bar{k}$ is injective.

2. If $\bar{j}$ is surjective and $\bar{\alpha}^{\prime}$ and $\bar{k}$ are injective then $\bar{i}$ is surjective.

Proof. (1) Assume $\eta(c) \in \operatorname{Ker} \bar{k}$. Then $\nu(b) \in \nu_{B}$ such that $\bar{\beta} \nu(b)=\eta(c)$ since $\bar{\beta}$ is surjective. Now, $0=\bar{k} \eta(c)$ as $\eta(c) \in \operatorname{Ker} \bar{k}$

$0=\bar{k} \bar{\beta}(\nu(b))$ as $\bar{\beta}$ is surjective

$=\bar{\beta}^{\prime} \bar{j}(\nu(b))$ since diagram is commutative

$=\bar{j}(\nu(b)) \in \operatorname{Ker} \bar{\beta}^{\prime}=\operatorname{Im} \bar{\alpha}^{\prime}$ since the second row is fuzzy exact. So, we can write $\bar{j}(\nu(b))+$ 
$\bar{\alpha}^{\prime}\left(\mu^{\prime}\left(a_{1}^{\prime}\right)\right)$

$$
\begin{aligned}
& \bar{j}(\nu(b))+\bar{\alpha}^{\prime} \bar{i}\left(\mu\left(a_{1}\right)=\bar{\alpha}^{\prime} \bar{i}\left(\mu\left(a_{2}\right)\right.\right. \\
& \bar{j}(\nu(b))+\bar{j} \bar{\alpha}\left(\mu\left(a_{1}\right)=\bar{j} \bar{\alpha}\left(\mu\left(a_{2}\right)\right.\right. \\
& =\bar{j}(\nu(b)) \in \operatorname{Im} \bar{j} \bar{\alpha} \\
& =\nu(b) \in \operatorname{Im} \bar{\alpha}
\end{aligned}
$$

as $\bar{j}$ is injective. Then, $\nu(b) \in \operatorname{Ker} \bar{\beta}$ since the first row is exact. Which implies $\bar{\beta} \nu(b)=0$. Thus, $\eta(c)=0$. Hence Ker $\bar{k}$ is injective as all elements lying in it are 0.

(2) Let $\mu^{\prime}\left(a^{\prime}\right) \in \mu_{A^{\prime}}^{\prime}$. Since $\bar{j}$ is surjective therefore for each $\nu^{\prime}\left(b^{\prime}\right) \in \nu_{B}^{\prime} \exists \nu(b) \in \nu_{B}$ such that $\bar{j}(\nu(b))=\nu^{\prime}\left(b^{\prime}\right)$. Now since $\bar{\alpha}^{\prime}$ is injective, we have $\bar{j}(\nu(b))=\bar{\alpha}^{\prime}\left(\mu^{\prime}\left(a^{\prime}\right) \in \operatorname{Im} \bar{\alpha}^{\prime}=\operatorname{Ker} \bar{\beta}^{\prime}\right.$ as the second row is fuzzy exact. Now,

$=\bar{\beta}^{\prime} \bar{j}=\bar{k} \beta$ as the diagram is commutative

$=\bar{\beta}^{\prime} \bar{j}(\nu(b))=\bar{k} \bar{\beta}(\nu(b))$

$\Rightarrow \bar{k} \bar{\beta}(\nu(b))=0$ since $\bar{j}(\nu(b))$ belongs to Ker $\bar{\beta}^{\prime}$

$\Rightarrow \bar{\beta}(\nu(b)) \in \operatorname{Ker} \bar{k}$

where since $\bar{k}$ is injective, it implies $\bar{\beta}(\nu(b))=0$ gives rise to $\nu(b) \in \operatorname{Ker} \bar{\beta}$

$\Rightarrow \nu(b) \in \operatorname{Im} \bar{\alpha}$ \{Since the first row is fuzzy exact.\}

Then $\nu(b)+\bar{\alpha}\left(\mu\left(a_{1}\right)\right)=\bar{\alpha}\left(\mu\left(a_{2}\right)\right)$ for some $\mu\left(a_{1}\right)$ and $\mu\left(a_{2}\right) \in \mu_{A}$

Consider, $\bar{\alpha}^{\prime}\left(\mu^{\prime}\left(a^{\prime}\right)\right)=\bar{j}(\nu(b))=\bar{j}(\bar{\alpha}(\mu(a)))$

$=\bar{\alpha}^{\prime}(\bar{i}(\mu(a)))$ as the given diagram is commutative.

Hence, $\left.\mu^{\prime}\left(a^{\prime}\right)=\bar{i}(\mu(a))\right)$, since $\bar{\alpha}^{\prime}$ is injective. Therefore $\bar{i}$ is surjective.

Theorem 2.(5-Lemma) For a commutative diagramm of fuzzy $R$-semimodules having fuzzy exact rows, where $\mu_{i}$ are the fuzzy $R$-semimodules over $R$-semimodules $M_{i}$ and $\nu_{i}$ are over $N_{i}$

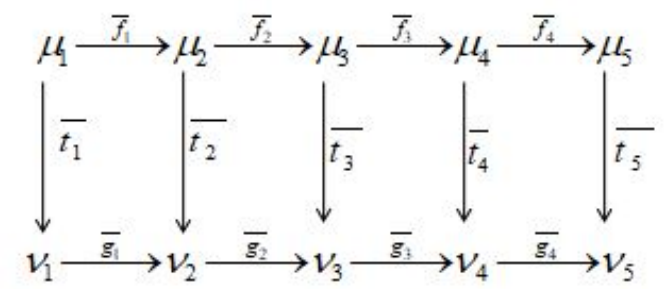

Fig.9 Commutativity fuzzy exact semimodule homomorphisms following stands true

1. If $\bar{t}_{2}$ and $\bar{t}_{4}$ are surjective and $\bar{t}_{5}$ is injective then $\bar{t}_{3}$ is surjective.

2. If $\bar{t}_{2}$ and $\bar{t}_{4}$ are injective and $\bar{t}_{1}$ is surjective, then $\bar{t}_{3}$ is injective.

3. If $\bar{t}_{1}, \bar{t}_{2}, \bar{t}_{4}$ and $\bar{t}_{5}$ are isomorphisms, then $\bar{t}_{3}$ is an isomorphism.

Proof. (1) let $\nu_{3}\left(n_{3}\right) \in \nu_{3} \exists \mu_{4}\left(m_{4}\right) \in \mu_{4}$ with $\bar{t}_{4}\left(\mu_{4}\left(m_{4}\right)\right)=\bar{g}_{3} \nu_{3}\left(n_{3}\right)$ [since $\bar{t}_{4}$ is surjective] $\Longrightarrow \bar{g}_{4} \bar{g}_{3} \nu_{3}\left(n_{3}\right)=0=\bar{g}_{4} \bar{t}_{4} \mu_{4}\left(m_{4}\right)=\bar{t}_{5} \bar{f}_{4} \mu_{4}\left(m_{4}\right)$ [since diagram is commutative]

$\Longrightarrow \bar{f}_{4} \mu_{4}\left(m_{4}\right)=0$ [since $\bar{t}_{5}$ is injective]

$\Longrightarrow \exists \mu_{3}\left(m_{3}\right) \in \mu_{3}$ with $\bar{f}_{3}\left(\mu_{3}\left(m_{3}\right)\right)=\mu_{4}\left(m_{4}\right)$.

Consider,

$$
\begin{aligned}
& \bar{g}_{3}\left(\nu_{3}\left(n_{3}\right)-\bar{t}_{3}\left(\mu_{3}\left(m_{3}\right)\right)=\bar{g}_{3}\left(\nu_{3}\left(n_{3}\right)-\bar{g}_{3} \bar{t}_{3}\left(\mu_{3}\left(m_{3}\right)\right)\right.\right. \\
& =\bar{t}_{4}\left(\mu_{4}\left(m_{4}\right)\right)-\bar{t}_{4} \bar{f}_{3}\left(\mu_{3}\left(m_{3}\right)\right) \\
& =\bar{t}_{4}\left(\mu_{4}\left(m_{4}\right)\right)-\bar{t}_{4}\left(\mu_{4}\left(m_{4}\right)\right) \\
& =0
\end{aligned}
$$

$\Longrightarrow \exists \nu_{2}\left(n_{2}\right) \in \nu_{2}$ with $\bar{g}_{2}\left(\nu_{2}\left(n_{2}\right)\right)=\left(\nu_{3}\left(n_{3}\right)-\right.$ $\bar{t}_{3}\left(\mu_{3}\left(m_{3}\right)\right)$

$\Longrightarrow \exists\left(\mu_{2}\left(m_{2}\right)\right) \in \mu_{2}$ with $\bar{t}_{2}\left(\mu_{2}\left(m_{2}\right)\right)=\nu_{2}\left(n_{2}\right)$ [since $\bar{t}_{2}$ is surjective]

Then

$$
\begin{array}{r}
\bar{t}_{3}\left[\bar{f}_{2}\left(\mu_{2}\left(m_{2}\right)\right)+\mu_{3}\left(m_{3}\right)\right]=\bar{t}_{3}\left(\bar{f}_{2} \mu_{2}\left(m_{2}\right)\right)+ \\
\bar{t}_{3}\left(\mu_{3}\left(m_{3}\right)\right) \\
=\bar{g}_{2} \bar{t}_{2}\left(\mu_{2}\left(m_{2}\right)\right)+\bar{t}_{3}\left(\mu_{3}\left(m_{3}\right)\right) \\
=\bar{g}_{2}\left(\nu_{2}\left(n_{2}\right)\right)+\bar{t}_{3}\left(\mu_{3}\left(m_{3}\right)\right) \\
=\left(\nu_{3}\left(n_{3}\right)\right)-\bar{t}_{3}\left(\mu_{3}\left(m_{3}\right)\right)+\bar{t}_{3}\left(\mu_{3}\left(m_{3}\right)\right) \\
=\left(\nu_{3}\left(n_{3}\right)\right)
\end{array}
$$

(2) Let $\mu_{3}\left(m_{3}\right) \in \mu_{3}$ with $\bar{t}_{3}\left(\mu_{3}\left(m_{3}\right)\right)=0$ 
$\Longrightarrow \bar{t}_{4} \bar{f}_{3}\left(\mu_{3}\left(m_{3}\right)\right)=\bar{g}_{3} \bar{t}_{3}\left(\mu_{3}\left(m_{3}\right)\right)=0$

$=\bar{f}_{3}\left(\mu_{3}\left(m_{3}\right)\right)=0\left[\bar{t}_{4}\right.$ injective $]$

$=\exists\left(\mu_{2}\left(m_{2}\right)\right) \in \mu_{2}$ with $\bar{f}_{2}\left(\mu_{2}\left(m_{2}\right)\right)=\left(\mu_{3}\left(m_{3}\right)\right)$

$=\bar{g}_{2} \bar{t}_{2}\left(\mu_{2}\left(m_{2}\right)\right)=\bar{t}_{3} \bar{f}_{2}\left(\mu_{2}\left(m_{2}\right)\right)=\bar{t}_{3}\left(\mu_{3}\left(m_{3}\right)\right)=0$

$=\exists\left(\nu_{1}\left(n_{1}\right)\right) \in \nu_{1}, \bar{g}_{1}\left(\nu_{1}\left(n_{1}\right)\right)=\bar{t}_{2}\left(\mu_{2}\left(m_{2}\right)\right)$

$=\exists\left(\mu_{1}\left(m_{1}\right)\right) \in \mu_{1}, \bar{t}_{1}\left(\mu_{1}\left(m_{1}\right)\right)=\left(\nu_{1}\left(n_{1}\right)\right)$

$=\bar{g}_{1} \bar{t}_{1}\left(\mu_{1}\left(m_{1}\right)\right)=\bar{g}_{1}\left(\nu_{1}\left(n_{1}\right)\right)$

$=\bar{t}_{2}\left(\bar{f}_{1}\left(\mu_{1}\left(m_{1}\right)\right)\right)=\bar{t}_{2}\left(\mu_{2}\left(m_{2}\right)\right)$

$=\bar{f}_{1}\left(\mu_{1}\left(m_{1}\right)=\left(\mu_{2}\left(m_{2}\right)\right)\right.$

$=\bar{f}_{2}\left(\mu_{2}\left(m_{2}\right)=\bar{f}_{2} \bar{f}_{1}\left(\mu_{1}\left(m_{1}\right)\right)=0=\left(\mu_{3}\left(m_{3}\right)\right)\right.$.

Thus $\bar{t}_{3}$ is injective.

(3) Trivial.

Proposition 2. Let $\bar{h}: \eta_{P} \rightarrow \nu_{Q}$ be a homomorphism of fuzzy $R$-semimodules. The following are equivalent:

(i) $\bar{h}$ is injective.

(ii) $\bar{h}$ is K-regular and $0 \rightarrow \eta_{P} \stackrel{\bar{h}}{\rightarrow} \nu_{Q}$ is fuzzy exact.

(iii) $\bar{h}$ is K-regular with Ker $\bar{h}=0$.

(iv) $\bar{h}$ is K-regular semi monomorphism.

(v) $\bar{h}$ is a monomorphism.

Proof. (i) $\Rightarrow$ (ii) Let $\bar{h}$ is injective. Let $\eta(p)$ and $\eta\left(p^{\prime}\right)$ be in $\eta_{P}$ such that $\bar{h}(\eta(p))=\bar{h}\left(\eta\left(p^{\prime}\right)\right)$ then $(\eta(p))=\left(\eta\left(p^{\prime}\right)\right)$ hence $(\eta(p))+0=\left(\eta\left(p^{\prime}\right)\right)+0$ with $0 \in \operatorname{Ker} \bar{h}$. Hence $\bar{h}$ is K-regular. Recall that $\bar{h}: \eta_{P} \rightarrow \nu_{Q}$ is fuzzy exact if and only if $\operatorname{Im}(0)=$ $\operatorname{Ker} \bar{h}=0$.

$\eta(p) \in \operatorname{Ker} \bar{h} \Rightarrow \bar{h}(\eta(p))=0=\bar{h}(0)$. Then $\eta(p)$ $=0$ because $\bar{h}$ is injective. Thus, Ker $\bar{h}=0$ and the sequence is fuzzy exact.

(ii) $\Rightarrow$ (iii) Trivial

(iii) $\Rightarrow$ (iv) Trivial

(iv) $\Rightarrow$ (v) Let $\bar{h}$ is K-regular semi monomorphism. Let $\bar{\gamma}_{1}: \mu_{A} \rightarrow \eta_{P}$ and $\bar{\gamma}_{2}: \mu_{A} \rightarrow \eta_{P}$ be the two homomorphism of fuzzy R-semimodules such that $\bar{h} o \bar{\gamma}_{1}=\bar{h} o \bar{\gamma}_{2} \ldots \ldots .(1)$.

Since $\bar{h}$ is K-regular so $\exists \bar{h}\left(\eta\left(p_{1}\right)\right)=\bar{h}\left(\eta\left(p_{2}\right)\right)$ such that $\eta\left(p_{1}\right)+\eta\left(p_{3}\right)=\eta\left(p_{2}\right)+\eta\left(p_{4}\right)$ for some $\eta\left(p_{3}\right)$ and $\eta\left(p_{4}\right) \in \operatorname{Ker} \bar{h}$. From equation 1 we have $\bar{h} o \bar{\gamma}_{1}(\mu(a))=\bar{h} o \bar{\gamma}_{2}(\mu(a))$

$$
\begin{aligned}
& \bar{h}\left[\bar{\gamma}_{1}(\mu(a))\right]=\bar{h}\left[\bar{\gamma}_{2}(\mu(a))\right] \\
& =\left[\bar{\gamma}_{1}(\mu(a))\right]+K=\left[\bar{\gamma}_{2}(\mu(a))\right]+K^{\prime} \\
& =\left[\bar{\gamma}_{1}(\mu(a))\right]=\left[\bar{\gamma}_{2}(\mu(a))\right] \\
& =\left[\bar{\gamma}_{1}\right]=\left[\bar{\gamma}_{2}\right]
\end{aligned}
$$

Thus, $\bar{h}$ is a monomorphism.

(v) $\Rightarrow$ (i) Let $\bar{h}: \eta_{P} \rightarrow \nu_{Q}$ be the monomorphism such that $\bar{h} o \bar{\gamma}_{1}=\bar{h} o \bar{\gamma}_{2} \Rightarrow \bar{\gamma}_{1}=\bar{\gamma}_{2}$ where $\bar{\gamma}_{1}: \mu_{Z} \rightarrow \eta_{P}$ and $\bar{\gamma}_{2}: \mu_{Z} \rightarrow \eta_{P}$.

We need to show $\bar{h}$ is injective

Let $\bar{h}\left(\eta\left(p_{1}\right)=\bar{h}\left(\eta\left(p_{2}\right)\right.\right.$. Assume $\bar{h}$ is not injective then $\exists \eta\left(p_{1}\right)$ and $\eta\left(p_{2}\right) \in \eta_{P}$ such that $\eta\left(p_{1}\right) \neq \eta\left(p_{2}\right) \Rightarrow \bar{h}\left(\eta\left(p_{1}\right)=\bar{h}\left(\eta\left(p_{2}\right)\right.\right.$

Let $P_{1}=\left[\eta\left(p_{1}\right), \eta\left(p_{2}\right)\right]$. Define $\eta_{1}: P_{1} \rightarrow[0,1]$ by $\eta_{1}\left(P_{1}\right)=\eta\left(p_{i}\right)$ for all $i=1,2$.

Define $\quad \bar{\gamma}_{1}: \eta_{1} \quad \rightarrow \quad \eta_{P} \quad$ as $\quad \bar{\gamma}_{1}\left[\eta_{1}\left(\eta\left(p_{1}\right)\right)\right]$ $=\bar{\gamma}_{1}\left[\eta_{1}\left(\eta\left(p_{2}\right)\right)\right]=\eta\left(p_{1}\right)$ and $\bar{\gamma}_{2}\left[\eta_{1}\left(\eta\left(p_{1}\right)\right)\right]=$ $\bar{\gamma}_{2}\left[\eta_{1}\left(\eta\left(p_{2}\right)\right)\right]=\eta\left(p_{2}\right)$. Since $\bar{h}$ is fuzzy monomorphism we have $\bar{h} o \bar{\gamma}_{1}=\bar{h} o \bar{\gamma}_{2} \Rightarrow \bar{\gamma}_{1}=\bar{\gamma}_{2}$

$$
\Rightarrow \eta\left(p_{1}\right)=\eta\left(p_{2}\right) \text {. }
$$

which is a contradiction to equation (1) above. Conversely, Let $\bar{h}$ is injective and we need to show $\bar{h}$ is monomorphism. For the same let us assume $\bar{h} o \bar{\gamma}_{1}=\bar{h} o \bar{\gamma}_{2}$ and we need to prove $\bar{\gamma}_{1}=\bar{\gamma}_{2}$ where $\bar{\gamma}_{1}: \mu_{Z} \rightarrow \eta_{P}$ and $\bar{\gamma}_{2}: \mu_{Z} \rightarrow \eta_{P}$.

$$
\begin{aligned}
& \bar{h} o \bar{\gamma}_{1}(\mu(z))=\bar{h} o \bar{\gamma}_{2}(\mu(z)) \\
& =\bar{h}\left[\bar{\gamma}_{1}(\mu(z))\right]=\bar{h}\left[\bar{\gamma}_{2}(\mu(z))\right] \\
& =\bar{h}\left(\eta\left(p_{1}\right)\right)=\bar{h}\left(\eta\left(p_{2}\right)\right) \\
& =\left(\eta\left(p_{1}\right)\right)=\left(\eta\left(p_{2}\right)\right)
\end{aligned}
$$

Since $\bar{h}$ is injective. Hence $\bar{\gamma}_{1}=\bar{\gamma}_{2}$.

\section{Fuzzy Projective Semimodules}

In this section fuzzy projective semimodules via Hom functor are discussed. Precisely, Theorem 3 demonstrates when does a fuzzy semimodule $\mu_{P}$ is fuzzy projective. Also towards the end we have shown that, in semimodule theory every fuzzy free is fuzzy projective however the converse is true only with a specific situation.

Definition 8. A fuzzy R-semimodule $\mu_{P}$ is called 
projective if and only if for every surjective fuzzy $R$-homomorphism $\bar{f}: \mu_{A} \rightarrow \mu_{B}$ and for every fuzzy $R$-homomorphism $\bar{g}: \mu_{P} \rightarrow \mu_{B}$ there exist a fuzzy $R$-homomorphism $\bar{h}: \mu_{P} \rightarrow \mu_{A}$ such that the figure below commutes that is : $\bar{f} \bar{h}=\bar{g}$.

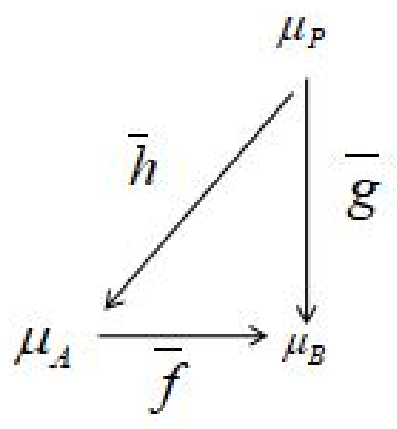

Fig.10 Fuzzy projective semimodule

Theorem 3. The following are comparable statements concerning fuzzy left $R$ - semimodules $\mu_{P}$ :

(i) $\mu_{P}$ is fuzzy projective

(ii) There is always a fuzzy proper exact induced sequence of homomorphism $0 \rightarrow \operatorname{hom}_{R}\left(\mu_{P}\right.$, $\left.\mu_{M^{\prime}}\right) \stackrel{\bar{\alpha}^{\prime}}{\rightarrow} \operatorname{hom}_{R}\left(\mu_{P}, \nu_{M}\right) \stackrel{\bar{\beta}^{\prime}}{\rightarrow} \operatorname{hom}_{R}\left(\mu_{P}\right.$, $\left.\eta_{M^{\prime \prime}}\right) \rightarrow 0$ for any fuzzy proper exact sequence

$\mu_{M^{\prime}} \stackrel{\bar{\alpha}}{\rightarrow} \nu_{M} \stackrel{\bar{\beta}}{\rightarrow} \eta_{M^{\prime \prime}}$ with $\bar{\beta}$ being K-regular.

Proof. (ii) $\Rightarrow$ (i) Suppose $\bar{\alpha}: \quad \mu_{N} \rightarrow \nu_{M}$ be surjective. As $\mu_{N} \stackrel{\bar{\alpha}}{\rightarrow} \nu_{M} \rightarrow 0$ is fuzzy proper exact sequence with $\nu_{M} \rightarrow 0$ being regular, it implies (ii) $\operatorname{hom}_{R}\left(\mu_{P}, \mu_{N}\right) \rightarrow \operatorname{hom}_{R}\left(\mu_{P}, \mu_{M}\right) \rightarrow 0$ is proper fuzzy exact sequence. Which further concludes $\mu_{P}$ is projective. Since every fuzzy proper exact is exact, and by Theorem 3.4[22]

(i) $\Rightarrow$ (ii) Let us assume $\mu_{P}$ to be fuzzy projective. and $\mu_{M} \stackrel{\vec{\alpha}}{\rightarrow} \nu_{M} \stackrel{\bar{\beta}}{\rightarrow} \eta_{M^{\prime \prime}}$ as a fuzzy proper exact with $\bar{\beta}$ being K-regular. Consider the sequence $0 \rightarrow \operatorname{ker} \bar{\beta} \stackrel{\bar{\theta}}{\rightarrow} \nu_{M} \stackrel{\bar{\phi}}{\rightarrow} \nu_{M} / \operatorname{ker} \bar{\beta} \rightarrow 0$ where $\bar{\phi}(\nu(m))$ $=\nu(m) / \operatorname{Ker} \bar{\beta}$ is canonical surjection. Then, $\bar{\theta}$ is injective. As we have $\mu_{P}$ fuzzy projective the sequence $0 \rightarrow \operatorname{hom}_{R}\left(\mu_{P}, \operatorname{Ker} \bar{\beta}\right) \stackrel{\bar{\theta}^{\prime}}{\rightarrow} \operatorname{hom}_{R}$ $\left(\mu_{P}, \nu_{M}\right) \stackrel{\bar{\phi}^{\prime}}{\rightarrow} h^{\prime} m_{R}\left(\mu_{P}, \nu(m) / \operatorname{Ker} \bar{\beta}\right) \rightarrow 0$ is fuzzy proper exact sequence. Define $\bar{g}: \mu_{M^{\prime}} \rightarrow \operatorname{Ker} \bar{\beta}$ and $\bar{f}: \quad\left(\nu_{M}\right) / \operatorname{Ker} \bar{\beta} \rightarrow \eta_{M^{\prime \prime}}$ where $\bar{g}\left(\mu\left(m^{\prime}\right)\right)$ $=\bar{\alpha}\left(\mu\left(m^{\prime}\right)\right)$ and $\bar{f}(\nu(m) / \operatorname{Ker} \bar{\beta})=\bar{\beta}(\nu(m))$. Let $\bar{f}(\nu(m) / \operatorname{Ker} \bar{\beta})=\bar{f}\left(\mu\left(m^{\prime}\right) / \operatorname{Ker} \bar{\beta}\right)$ then $\bar{\beta}(\nu(m))$ $=\bar{\beta}\left(\mu\left(m^{\prime}\right)\right)$. Since $\bar{\beta}$ is K-regular then $\nu(m)+$ $\nu\left(m_{1}\right)=\mu\left(m^{\prime}\right)+\nu\left(m_{2}\right)$ where $\nu\left(m_{1}\right)$ and $\nu\left(m_{2}\right)$ $\in \operatorname{Ker} \bar{\beta}$. Hence $(\nu(m)) / \operatorname{Ker} \bar{\beta}=$ $\left(\mu\left(m^{\prime}\right) / \operatorname{Ker} \bar{\beta}\right.$ therefore $\bar{f}$ is injective. Taking into account the commutative diagram below

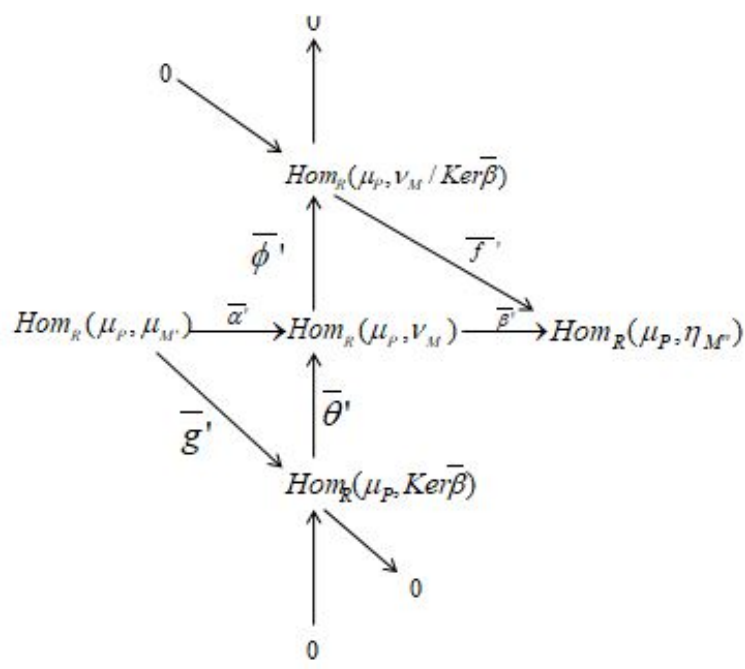

Fig.11 Commutative induced sequence of homomorphism

where $\bar{g}^{\prime}(\bar{\rho})=\bar{g}(\bar{\rho})$ and $\bar{f}^{\prime}(\bar{\lambda})=\bar{g}(\bar{\lambda})$ where $\bar{\rho} \in$ $\operatorname{Hom}_{R}\left(\mu_{P}, \mu_{M^{\prime}}\right)$ and $\bar{\lambda} \in \operatorname{Hom}_{R}\left(\mu_{P}, \nu_{M} / \operatorname{Ker} \bar{\beta}\right)$. Now Let $\bar{\rho}, \bar{\lambda} \in \operatorname{Hom}_{R}\left(\mu_{P}, \nu_{M} / \operatorname{Ker} \bar{\beta}\right)$ such that $\bar{f}^{\prime}(\bar{\rho})=\bar{f}^{\prime}(\bar{\lambda})$. Since $\bar{f}^{\prime}$ is injective then $\bar{\rho}=\bar{\lambda}$. Let $\bar{\rho} \in \operatorname{Hom}_{R}\left(\mu_{P}, \operatorname{Ker} \bar{\beta}\right)$. Since $\mu_{P}$ is fuzzy projective then there exist $\bar{\gamma}: \mu_{P} \rightarrow \mu_{M^{\prime}}$ such that the following figure commutes.

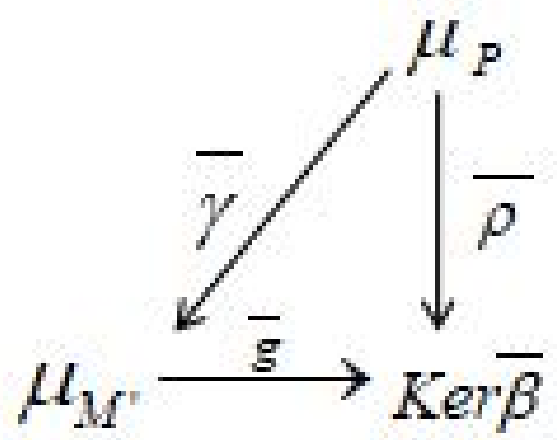

Fig.12 $\mu_{P}$ is fuzzy projective 
Thus $\bar{g}^{\prime}$ is surjective. Thus, the sequence $0 \rightarrow$ $\operatorname{hom}_{R}\left(\mu_{P}, \mu_{M^{\prime}}\right) \stackrel{\bar{\alpha}^{\prime}}{\rightarrow} \operatorname{hom}_{R}\left(\mu_{P}, \nu_{M}\right) \stackrel{\bar{\beta}^{\prime}}{\rightarrow}$ hom $_{R}$ $\left(\mu_{P}, \eta_{M^{\prime \prime}}\right) \rightarrow 0$ is fuzzy proper exact by corollary 2.

Corollary 4. The statements regarding fuzzy left $R$ - semimodules $\mu_{P}$ that follow are equivalent.

(i) $\mu_{P}$ is fuzzy projective

(ii) For every fuzzy proper exact sequence of left $R$-semimodules $\mu_{M^{\prime}} \stackrel{\bar{\alpha}}{\rightarrow} \nu_{M} \stackrel{\bar{\beta}}{\rightarrow} \eta_{M^{\prime \prime}}$ with $\bar{\beta}$ being regular. The induced sequence of homomorphisms

$0 \rightarrow \operatorname{hom}_{R}\left(\mu_{P}, \mu_{M^{\prime}}\right) \stackrel{\bar{\alpha}^{\prime}}{\rightarrow} \operatorname{hom}_{R}\left(\mu_{P}\right.$, $\left.\nu_{M}\right) \stackrel{\bar{\beta}^{\prime}}{\rightarrow} \operatorname{hom}_{R}\left(\mu_{P}, \eta_{M^{\prime \prime}}\right) \rightarrow 0$ is fuzzy proper exact.

Proof. It is a result of Theorem 3.

Proposition 3. Consider $\mu_{M}$, a fuzzy free

$R$-semimodule with a fuzzy basis $B$ and let $\nu_{N}$ be an arbitrary left fuzzy $R$-semimodule. For each function $\bar{g} \in \nu_{N}^{B}$ there is a unique fuzzy

$R$-homomorphism $\bar{\alpha}: \mu_{M} \rightarrow \nu_{N}$ satisfying $b \bar{\alpha}=$ $\bar{g}(b)$ for all $b \in B$.

Proof. As we know each element $\mu(m)$ of $\mu_{M}$ can be written in the form $\Sigma r_{b} b$ where $r_{b}$ are the elements of $\mathrm{R}$ where only a finite number of elements in $\mathrm{R}$ are not equal to zero. Define the function $\bar{\alpha}: \mu_{M} \rightarrow \nu_{N}$ by $\Sigma r_{b} b=\Sigma r_{b} g(b)$. It is trivial that $\bar{\alpha}$ is a homomorphism satisfying the requird property. Also, if $\bar{\beta}: \mu_{M} \rightarrow \nu_{N}$ is a R-homomorphism satisfying $\mathrm{b} \bar{\beta}=\bar{g}(b)$ for all $\mathrm{b} \in \mathrm{B}$ then $\left(\Sigma r_{b} b\right) \bar{\beta}$ $=\Sigma r_{b}(b \bar{\beta})=\Sigma r_{b} \bar{g}(b)=\Sigma r_{b} b \bar{\alpha}=\left(\Sigma r_{b} b\right) \bar{\alpha}$ thus, $\bar{\beta}=\bar{\alpha}$ implying $\bar{\alpha}$ is unique.

Proposition 4. Every fuzzy free R-semimodule is fuzzy projective.

Proof. Let $\mu_{P}$ be a fuzzy free R-semimodule having a fuzzy basis B. Let $\bar{\phi}: \eta_{M} \rightarrow \nu_{N}$ be a surjective R-homomorphism of fuzzy left Rsemimodules and let $\bar{\alpha}: \mu_{P} \rightarrow \nu_{N}$ be a Rhomomorphism. Since $\bar{\phi}$ is surjective for a element $\mathrm{b}$ of $\mathrm{B} \exists$ an element $\eta(m)$ of $\eta_{M}$ such that $\eta(m) \bar{\phi}=\mathrm{b} \bar{\alpha}$ from the proposition 3 we have $\bar{\beta}: \mu_{P} \rightarrow \eta_{M}$ satisfying $b \bar{\beta}=\eta(m)$. Then, $\bar{\alpha} \bar{\beta} \bar{\phi}=$ $\mu(m) \bar{\phi}=\mathrm{b} \bar{\alpha}$ for all $\mathrm{b} \in \mathrm{B}$. Also by the uniqueness part used in proposition 3 we have $\bar{\alpha}=\bar{\beta} \bar{\phi}$.
Proposition 5. A fuzzy left semimodule is fuzzy projective if and only if it is a retract of a fuzzy free left R-semimodule.

NOTE A left R-semimodule $\mu_{A}$ is a retract of a left R-semimodule $\mu_{B}$ if and only if there exist a surjective R-homomorphism $\bar{h}: \mu_{A} \rightarrow \mu_{B}$ and an R-homomorphism $\bar{g}: \mu_{B} \rightarrow \mu_{A}$ satisfying the condition that $\bar{g} \bar{h}$ is a identity map on $\mu_{B}$.

Proof. From the proof given in [13] we have $\overline{0} \rightarrow$ $\mu_{A} \stackrel{\bar{f}}{\rightarrow} \mu_{B} \stackrel{\bar{g}}{\rightarrow} \theta_{p} \rightarrow \overline{0}$ be an exact sequence where $\theta_{p}$ is fuzzy projective and $\mu_{B}$ is fuzzy free Rsemimodule, then in the following diagram, we can have

R-homomorphism $\bar{g}^{\prime}: \theta_{p} \rightarrow \mu_{B}$ to have a commutative diagram.

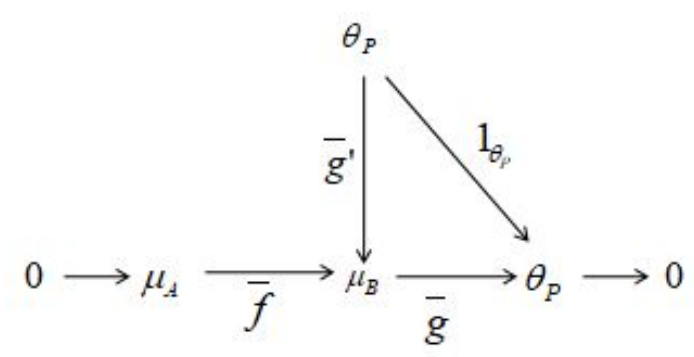

Fig.13 Commutativity is shown for the fuzzy projective $\theta_{p}$

Then $\bar{g} \bar{g}^{\prime}=1_{\theta_{p}}$. That is $\theta_{p}$ is retract of fuzzy free left R-semimodule $\mu_{B}$.

Conversely, let $\mu_{P}$ is retract of fuzzy free R-semimodules $\nu_{F}$ and let $\bar{\theta}: \nu_{F} \rightarrow \mu_{P}$ and $\bar{\psi}: \mu_{P} \rightarrow \nu_{F}$ be a R-homomorphisms such that $\bar{\theta}$ is surjective and $\bar{\psi} \bar{\theta}$ is identity map on $\mu_{P}$. Let $\bar{\phi}: \eta_{M} \rightarrow \pi_{N}$ be a surjective R-homomorphisms and let $\bar{\alpha}: \mu_{P} \rightarrow \pi_{N}$ be a R-homomorphisms. Since $\nu_{F}$ is fuzzy projective (by proposition 4 ), $\exists$ an R-homomorphisms $\bar{\beta}: \nu_{F} \rightarrow \eta_{M}$ such that $\bar{\beta} \bar{\phi}=\bar{\theta} \bar{\alpha}$. Thus, $\bar{\psi} \bar{\beta} \bar{\phi}=\bar{\psi} \bar{\theta} \bar{\alpha}=\bar{\alpha}$. Hence $\bar{\psi} \bar{\beta}: \mu_{P} \rightarrow \eta_{M}$ shows the required commutativity to prove the projectivity. This converse part is supported using the following figure. 


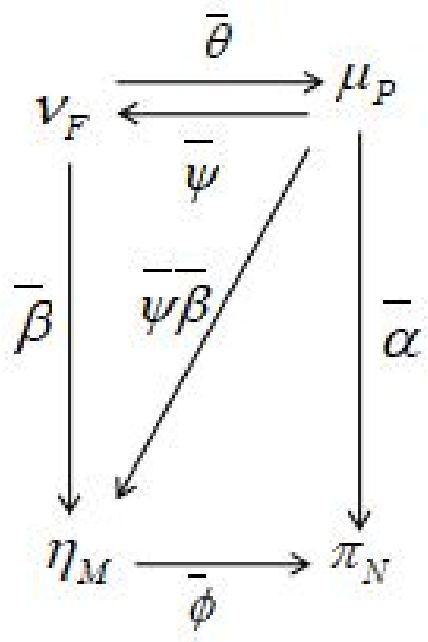

Fig.14 Exhibiting the commutativity for proving fuzzy projectivity

Result 3. $\mu_{P}$ is fuzzy projective if it is a direct summand of a fuzzy free semimodule $\mu_{F}$.

Proof. Let $\mu_{F} \cong \mu_{K} \oplus \mu_{P}$ and let $\bar{\pi}$ be the map $\mu_{F} \cong \mu_{K} \oplus \mu_{P} \rightarrow \mu_{P}$ where the second mapping is canonical projection. Similarly, let $\bar{h}$ be the map $\mu_{P} \rightarrow \mu_{F} \cong \mu_{K} \oplus \mu_{P}$ where the first mapping is canonical injection. Consider the following figure with horizontal row being fuzzy exact

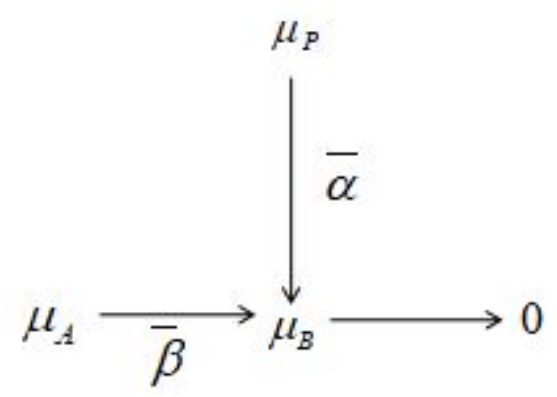

Fig.15 Horizontal row being exact

then in the figure given below

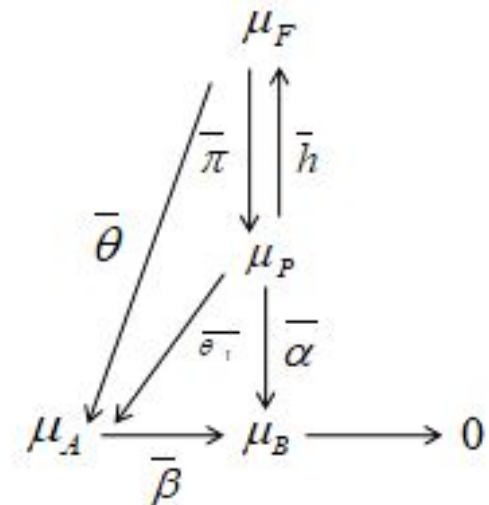

Fig.16 Exhibiting the fuzzy projectivity of $\mu_{P}$

Since $\mu_{F}$ is fuzzy projective we can have a homomorphism $\bar{\theta}: \mu_{F} \rightarrow \mu_{A}$ such that $\bar{\beta} \bar{\theta}=\bar{\alpha} \bar{h}$. Assume $\bar{\theta}_{1}=\bar{\theta} \bar{h}: \mu_{P} \rightarrow \mu_{A}$. Then $\bar{\beta} \bar{\theta}_{1}=$

$$
\begin{aligned}
& (\bar{\beta} \bar{\theta}) \bar{h}=(\bar{\alpha} \bar{\pi}) \bar{h} \\
& =\bar{\alpha}(\bar{\pi} \bar{h}) \\
& =\bar{\pi} 1_{\mu_{P}} \text { by proposition } 5 \\
& =\bar{\pi} .
\end{aligned}
$$

hence $\mu_{P}$ is fuzzy projective.

\section{Applications and future scope}

Apart from the areas of modern science[19], semirings and semimodules have given numerous applications in various branches of mathematics and computer science. Complete understanding of semimodules help us to understand the algebraic structure "semirings" and can result in applications not only in the fields of automata and optimization theory but also in generalizing fuzzy computation and bounded distributive lattices[6]. As we know, to comprehend ring $\mathrm{R}$ one must be aware of the fact that how $\mathrm{R}$ acts on two of its important substructures right and left modules. Thus, the module theory plays a crucial role in ring theory. The major subsections of module theory are projective and injective modules. Semimodules over semirings are the generalization of such algebraic structures and thereby help in broadening the theory of projective and injective modules into semimodules. To add in the existing literature, here in this paper we have 
given a new direction to the theory of semimodules and projective semimodules by giving it an extension in its fuzzy domain, which further can set platform for many novel researches. That is, it creates the staging to extend the research mentioned in [4], [8], [12], [13], [18], [22] to their corresponding fuzzy semimodule environment. Also, the present work encourages one to extend the current study to the fuzzy environment of the research mentioned in [10] so as to be useful in theory of fuzzy weighted automata [15], and to give an algebraic approach to fuzzy compression algorithms and reconstruction of digital images [11].

And can set a platform to define general algebraic frameworks for shortest-distance problems which are based on the structure of fuzzy semirings, including their fuzzy pseudocode. Last but not the least since the mathematical logic has evolved from the set theory concept. In fact, the entire digital world has evolved from the set theory, this work can thus opens door to future advances like [13].

\section{Conclusion}

Along with the generalization of well known results of semimodule theory, complete characterization of fuzzy projective semimodules is discussed here via Hom functor. Also, equivalent conditions in homomorphisms of fuzzy semimodules are touched during the study in addition to which the necessary condition for a fuzzy semimodule to be a fuzzy projective semimodule is explained with a specific condition.

\section{References}

[1] Ahsan, J., Saifullah, K. and Khan, M.F. Fuzzy Semirings, Fuzzy Sets and Systems, Vol.60, No.3, 1993, pp. 309-320.

[2] B. Khayut, L. Fabri, M. Avikhana, The Reasonable and Conscious Understanding System of reality Under Uncertainty,International journal of circuits, systems and signal processing, Vol. 14, 2020 pp. 296-308.
[3] Goguen, J.A. L-fuzzy sets, Journal of mathematical analysis and applications, Vol.18, No.1, 1967, pp. 145-174.

[4] Golan, J.S. Making modules fuzzy, Fuzzy Sets and Systems, Vol.32, No.1, 1989, pp. 91-94.

[5] Golan, J.S., The theory of semirings with applications in mathematics and theoretical computer science, Pitman Monographs and surveys in pure and applied Maths, no 54(Longman), New York, 1992.

[6] Golan, J.S., Semirings and Their Applications, Kluwer Academic Publishers, Dordrecht-Boston-London, 1999.

[7] Liu, W. Operations on fuzzy ideals, Fuzzy Sets and Systems, Vol.11, No.1, 1983, pp. 31-39.

[8] Liu, H.X. Fuzzy projective modules and tensor products in fuzzy module categories, Iranian Journal of Fuzzy Systems, Vol.11, No.2, 2014, pp. 89-101.

[9] Negoita, C.V. and Ralescu, D.A., Applications of fuzzy sets to systems Analysis, (Birkhauser) Basel, 1975.

[10] Nola, Di.A., Lenzi, G., Nam, T.G. and Vannucci, S. On injectivity of semimodules over additively idempotent division semirings and chain MV-semirings, Journal of Algebra, Vol.538, 2019, pp. 81-109.

[11] Nola, Di.A. and Russo, C. Łukasiewicz transform and its application to compression and reconstruction of digital images, Information Sciences, Vol.177, No.6, 2007, pp. 14811498.

[12] Pan, F. Fuzzy finitely generated modules, Fuzzy Sets and Systems, Vol.21, No.1, 1987, pp. 105-113.

[13] Osipov, A. On Some Fuzzy Classification Algorithms and the AEC Model, WSEAS Transactions on Systems, Vol.19, 2020, pp. 168-177.

[14] Satyanarayana, B., Godloza. L. and Mohiddin S.S. On fuzzy Dimension Of a module 
with DCC on submodules, Acharya Nagarjuna International Journal of Mathematics and Information Tech nology, Vol.1, No.1, 2004, pp. 13-32.

[15] Schwarz, S. Lukasiewicz logic and weighted logics over MV-semirings, Journal of Automata Languages and Combinatorics, Vol.12, No.4, 2007, pp. 485-499.

[16] Shu, Q.Y. and Wang X.P. The cardinality of bases in semilinear spaces over commutative semirings, Linear Algebra and its Applications, Vol.459, 2014, pp. 83-100.

[17] Shu, Q.Y. and Wang, X.P. Dimensional formulas of semilinear subspaces over semirings, Linear and Multilinear Algebra, Vol.62, No.11, 2014, pp. 1475-1490.

[18] Sahni, A.K., Pandey, J.T., Mishra, R.K. and Sinha V. K. Fuzzy Projective-Injective Modules and Their Evenness Over Semi-Simple Rings, Turkish Journal of Computer and Mathematics Education, Vol.12, No.6, 2021, pp. 3624-3634.

[19] Vandiver, H.S. Note on a simple type of Algebra in cancellation law does not hold, Bulletin of the American Mathematical Society, Vol.40, No.12, 1934, pp. 914-920.

[20] Xiaoyong, Z. and Hua, Z. A Lean Green Implementation Evaluation Method based on Fuzzy Analytic Net Process and Fuzzy Complex Proportional Assessment, International journal of circuits, systems and signal processing, Vol. 14, 2020, pp. 646-655.

[21] Zadeh, L.A. Fuzzy sets, Information and Control, Vol.8, No.3, 1965, pp. 338-353.

[22] Zahedi, M.M. and Ameri, R. On fuzzy projective and injective modules, The Journal of Fuzzy Mathematics, Vol.3, No.1, 1995, pp. 181-190 .

Contribution of individual authors to the creation of a scientific article (ghostwriting policy)

\section{Author Contributions:}

Amarjit kaur sahni formulated the manuscript after the necessary literature review. Constructed the examples, lemmas, theorems given and developed the procedure of fuzzy proper exact sequences.

Jayanti Tripathi Pandey extended the idea of fuzzy projective modules to fuzzy projective semimodules over semirings and proposed Theorem 3 mentioned in section 4 which is also one of main highlights of the paper, structured the abstract and examined the overall framing of the manuscript by suggesting valuable corrections.

Ratnesh Kumar Mishra proposed the idea of fuzzy projective modules.

Vinay Kumar cross verified all the constructed examples.

Sources of funding for research presented in a scientific article or scientific article itself

No fund

Creative Commons Attribution License 4.0 (Attribution 4.0 International, CC BY 4.0)

This article is published under the terms of the Creative Commons Attribution License 4.0

https : //creativecommons.org/li

censes/by/4.0/deed.en ${ }_{U} S$ 